\title{
Sarah AL-MATARY, La Haine des clercs. L'anti- intellectualisme en France
}

\section{Stéphanie Lanfranchi}

\section{(2) OpenEdition}

1 Journals

Édition électronique

URL : https://journals.openedition.org/ress/7282

DOI : $10.4000 /$ ress. 7282

ISBN : $1663-4446$

ISSN : 1663-4446

Éditeur

Librairie Droz

Édition imprimée

Date de publication : 21 mai 2021

Pagination : 276-280

ISSN : 0048-8046

Référence électronique

Stéphanie Lanfranchi, "Sarah AL-MATARY, La Haine des clercs. L'anti-intellectualisme en France », Revue européenne des sciences sociales [En ligne], 59-1 | 2021, mis en ligne le 21 mai 2021, consulté le 25 mai 2021. URL : http://journals.openedition.org/ress/7282 ; DOI : https://doi.org/10.4000/ress.7282

Ce document a été généré automatiquement le 25 mai 2021

(c) Librairie Droz 


\title{
Sarah AL-MATARY, La Haine des clercs. L'anti-intellectualisme en France
}

\author{
Stéphanie Lanfranchi
}

\section{RÉFÉRENCE}

Sarah AL-MATARY, 2019, La Haine des clercs. L'anti-intellectualisme en France, Paris, Éditions du Seuil, 392 p.

1 Plusieurs images pourraient sans doute convenir pour décrire la structure forte et élégante du livre de Sarah Al-Matary. La Haine des clercs se lit d'abord comme on visite une imposante galerie de portraits qui retrace, tout au long de ses treize chapitres et des presque deux siècles d'histoire française qu'ils parcourent (de 1848 au mandat présidentiel de Nicolas Sarkozy), une vision complexe et diachronique de l'antiintellectualisme en France. On découvre ainsi une multitude de tableaux individuels, admirables de précision et de nuances, exprimant l'originalité des positions antiintellectuelles assumées le plus souvent par les lettrés eux-mêmes. Le lecteur pourrait même envisager une consultation ponctuelle de l'ouvrage, en s'aidant de l'index, pour y trouver les pages qui le renseigneraient, avec un esprit de synthèse qui ne sacrifie pourtant rien à la finesse de l'analyse et à la richesse des citations, sur les formes que l'anti-intellectualisme a prises chez un auteur ou dans une revue donnés. Mais ce n'était manifestement pas l'idée ni l'intention de l'autrice, qui nous invite plutôt à parcourir l'ensemble de sa galerie: elle décourage d'ailleurs quelque peu les lecteurs pressés ou en quête d'efficacité, qui voudraient se servir de la table des matières pour se rendre directement au chapitre qui les intéresse, car elle ne fournit aucun repère chronologique ni biographique dans ses titres et sous-titres.

Le tracé est pourtant presque parfaitement linéaire, et nous conduit tout au long des trois premiers chapitres à travers les polémiques qui façonnent la définition politique et sociale de l'intellectuel au XIX ${ }^{\mathrm{e}}$ siècle, dans son rapport antithétique - ou tout au moins problématique - avec le savoir-faire et le travail manuels (chap. 1) qui seraient 
propres aux classes populaires (chap. 2), et qui culminent dans les clivages pourtant mouvants de l'Affaire Dreyfus (chap.3). Les deux chapitres suivants distinguent les formes que le discours anti-intellectualiste prend, à l'aube du nouveau siècle, chez des anciens anti-dreyfusards tout comme chez certains de leurs adversaires. Aussi les premiers composent-ils une rhétorique de la décadence chère aux milieux maurassiens, assortie d'anti-féminisme, d'anti-romantisme et d'anti-républicanisme (chap. 4); alors que les seconds développent, notamment dans les milieux anarchistes et syndicalistes, un discours très critique à l'égard de l'école, de l'Université et de ses représentants, tout en proposant des modèles d'éducation alternatifs et populaires (chap. 5). Le chapitre consacré à la guerre (chap.6) analyse les ressorts anti-intellectualistes qu'exploite massivement la propagande pour vilipender, à l'extérieur, la «science allemande » et, à l'intérieur, la figure de l'embusqué ou de l'exempté. Sarah Al-Matary montre à quel point les traces laissées par cette rhétorique sont profondes et durables, y compris dans la perception que les intellectuels ont d'eux- mêmes, surtout lorsque l'expérience des tranchées révèle parallèlement leur vulnérabilité et souvent leur incompétence par rapport aux soldats issus des classes ouvrières et paysannes. L'antiintellectualisme apparaît comme une vague de fond au lendemain de la guerre, entraînant avec elle un antirationalisme et un antiparlementarisme structurels qui alimentent les positions les plus variées, et même opposées, durant l'entre-deuxguerres. Des maurassiens aux anti-maurassiens dans les rangs catholiques (chap. 7), des fascistes aux communistes (chap. 8), l'anti-intellectualisme envahit l'Europe et déferle sur la France aussi. La vague se nourrit des débats suscités par plusieurs événements clivants qui ponctuent l'actualité nationale et internationale de ces années-là, parmi lesquels ne figurent pas seulement l'essor des régimes totalitaires en Europe et la condamnation de l'Action française, mais aussi des faits de moindre envergure, telle que l'affaire de la Grande Chartreuse, dont l'autrice retrace très efficacement les accents et les enjeux anti-intellectualistes et anti-républicains. Dans les années 1930 et 1940, l'anti-intellectualisme français s'abreuve également à des sources étrangères, qu'elles soient russes ou allemandes (chap. 9). La dimension largement antisémite de l'anti-intellectualisme français, clairement identifiée dès le XIX siècle ("Tous les antiintellectualistes ne sont pas antisémites; reste que depuis Proudhon et Veuillot, la plupart des antisémites goûtent l'anti-intellectualisme", déclare Sarah Al-Matary, p. 94), éclate au grand jour de ce que l'autrice propose d'envisager comme « une crise nazie de la pensée française » (chap. 10). Les trois derniers chapitres, consacrés aux formes multiples que l'anti-intellectualisme a adoptées dans l'après-guerre, se concentrent d'abord sur la guerre d'Algérie et le poujadisme (chap.11), puis sur les évènements et les interprétations de Mai 68 (chap.12), pour aboutir à un dernier chapitre sur les premières années du XXI ${ }^{\mathrm{e}}$ siècle, après que Jean-Marie Le Pen a accédé au second tour des élections présidentielles. L'équilibre du discours se trouve profondément modifié dans cette dernière partie du livre, en ce qu'une part croissante est désormais consacrée à l'anti-intellectualisme des hommes politiques, plutôt qu'aux positions exprimées par les intellectuels eux-mêmes. Cela permet de mieux donner à voir l'émergence d'un anti-intellectualisme d'État que Sarah Al-Matary fait remonter non pas à Vichy, comme on aurait peut-être pu s'y attendre, mais aux «évènements d'Algérie » et aux déclarations d'un ministre socialiste, Maurice Bourgès-Maunoury. Elle en décrit ensuite l'inquiétante résurgence après 2002, au sommet de l'État comme sur les plateaux de télévision : c'est ainsi que réapparaissent les formules maintes fois rencontrées dans les pages précédentes du livre, que les stratégies de communication 
et les tentations populistes empruntent au vocabulaire et aux traditions de l'antiintellectualisme, sur fond de disparition de l'intellectuel de la sphère publique.

3 Ce n'est pas une moindre réussite de l'ouvrage de Sarah Al-Matary, que de se déployer comme une suite de tableaux pointillistes, tout en parvenant à nous offrir une vue d'ensemble d'une extrême cohérence. Cela s'avérait d'autant plus malaisé, qu'aucune définition précise ne se dégage pourtant en creux de l'intellectuel, si bien que la conclusion du livre dresse le constat d'une cible «mouvante » des anti-intellectualistes (p. 306), touchant, tour à tour, les gens de lettres, les artistes, les enseignants, mais aussi les fonctionnaires, les parlementaires ou les technocrates. En revanche, Sarah AlMatary parvient à déceler une certaine stabilité dans les « griefs » qu'on formule à leur égard, et même une récurrence: leur prétendue méconnaissance du réel, leur idéalisme, leur opportunisme, leur parasitisme, leur inutilité, voire leur nocivité. Par ailleurs, si son récit met à découvert certains tournants et moment de rupture, il saisit avant tout les continuités, les réseaux et les filiations, parfois improbables, d'un siècle à l'autre, entre les représentants les plus significatifs de cette haine des clercs. Elle suggère ainsi de lire «l'histoire de l'anti-intellectualisme [...] comme un roman avec retour de personnages ", tout en relevant que «l'exploration généalogique montre que les filiations directes sont rares, et tend à souligner la convergence d'apports divers. » (p. 309).

4 La nature même de l'anti-intellectualisme imposait sans doute, commente l'autrice, une approche «fortement personnalisé[e], de par sa nature polémique, mais aussi parce que ses hérauts fondent souvent leur réaction contre un groupe » et "sur la mise en scène d'une exceptionnalité - la leur ou celle d'un chef charismatique ». C'est un sujet qui se "prête au portrait» et dont la représentation tend d'autant plus à l'individualisation que l'on recherche les motifs profonds d'une haine inscrite dans la biographie et la personnalité de ces hommes. Cette méthodologie n'est pourtant pas exempte de risques, lorsqu'il s'agit de comprendre les raisons, parfois enfouies et refoulées, qui portent ces intellectuels à haïr des personnes et des typologies qui leur ressemblent. Mais l'autrice avance avec prudence et même avec une certaine discrétion, laissant au lecteur le soin de formuler ses propres jugements, ne cédant $\mathrm{ni}$ aux raccourcis psychologisants, ni au simplisme des schémas: "Pas plus que l'hypothèse compensatoire, l'hypothèse expiatoire ne rend compte de la complexité des situations » (p. 267), affirme-t-elle pour décrire les itinéraires des premiers «établis » maoïstes dans les années 1968. Cette remarque pourrait s'étendre à l'ensemble des portraits que Sarah Al-Matary a dressés dans son livre, tant elle a pris le soin systématique de pondérer les éléments qui relèvent d'un effet de groupe, de génération et de sociabilité, avec des considérations plus personnelles, notamment sur le parcours familial et scolaire de ces hommes. On ne résumera pas la complexité et la particularité irréductibles d'une bonne soixantaine de portraits en quelques lignes seulement. Citons seulement quelques perles de ce collier d'anti-intellectualisme : comme Proudhon, que l'on a trop souvent voulu peindre en autodidacte (chap. 1) ; comme Zola qui clamait son anti-intellectualisme avant d'incarner l'intellectuel dreyfusard (chap.3); comme Maurras à qui la surdité empêche de suivre les conférences de la Sorbonne (chap. 4); comme Robert Hertz décrivant dans les lettres du front à son épouse le désarroi de se découvrir inadapté, et l'espoir de concevoir ensemble une pédagogie nouvelle qui rapprocherait les élèves du peuple et de la nature (chap.6); comme Bernanos qui souffre, enfant, de voir son école catholique fermée par les décrets des années 1880 et voue ensuite une haine croissante aux "professeurs» qui peuplent ses romans 
(chap. 7) ; comme le Nizan normalien et anti-intellectualiste (chap. 6 et 8); comme Céline brouillant constamment les pistes pour passer sous silence ses études et ses lectures savantes (chap.9); comme Drieu la Rochelle et Aragon, dans leur rapport tumultueux et compétitif (chap.10); ou enfin comme Onfray et Houellebecq que les plateaux de télévision opposent pour créer le show, mais qui partagent le même mépris des intellectuels (chap. 13).

5 Après la galerie de portrait, l'image du collier, avec son enfilade de perles donnant chacune à voir des nuances et des miroitements particuliers tout en composant un ensemble, renvoie à un équilibre similaire entre l'objet individuel et collectif, mais y ajoute une référence plus explicite à sa préciosité. Or, les richesses que déploie ce livre font partie du plaisir qu'il procure : elles tiennent d'abord aux documents (même le lecteur sévère qui voudrait y déplorer l'absence d'archives ne pourra que reconnaître le foisonnement et la grande variété des sources éditées qui y sont exploitées et composent un corpus primaire et secondaire, sinon exhaustifs, du moins extrêmement larges et cohérents), et elles tiennent enfin à la langue. Sarah Al-Matary choisit un style littéraire, qui ne cède jamais au relâchement d'une prose journalistique, pas plus qu'il ne cède à la facilité de la pédanterie ou du jargon académique.

6 Au demeurant, bien que cela ne constitue en rien l'objet central de son travail, l'autrice révèle non seulement la permanence, du XIX ${ }^{e}$ au XXI siècle, d'une forte misogynie dans la plupart des pensées anti-intellectualistes françaises, mais aussi, presque a contrario, l'absence d'anti-intellectualisme chez les femmes intellectuelles «qui peinent à faire reconnaître leur capacité à penser » (p. 16). Le style que choisit l'autrice, cette langue exigeante et contrôlée, devient le geste politique d'une femme cultivée revendiquant sans ambages son statut de lettrée à l'époque où les intellectuels semblent avoir disparu du tableau alors que l'anti-intellectualisme fait plus que jamais rage.

\section{AUTEURS}

\section{STÉPHANIE LANFRANCHI}

ENS de Lyon - Triangle 